\title{
Patient, Nutzer, Kunde?
}

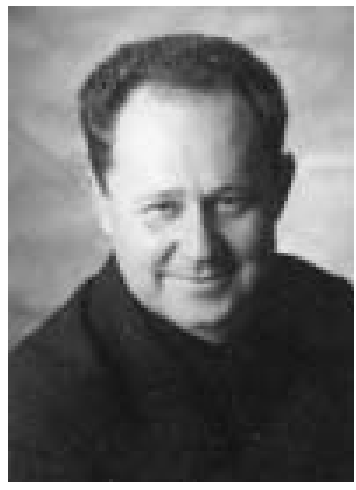

Prof. Dr. Manfred Wildner
Bibliografie

DOI http://dx.doi.org/

10.1055/s-0031-1277149

Gesundheitswesen 2011;

73: 397-398

(c) Georg Thieme Verlag KG

Stuttgart · New York

ISSN 0941-3790

Korrespondenzadresse

Prof. Dr. Manfred Wildner

Bayerisches Landesamt

für Gesundheit und

Lebensmittelsicherheit

Veterinärstraße 2

85762 Oberschleißheim

Manfred.Wildner@lgl.bayern.de
„Homo oeconomicus“ ist eine vergleichsweise junge Spielform der Spezies Mensch. Ob er tatsächlich existiert, ist ungewiss. In den Wirtschaftswissenschaften wird damit ein fiktiver Akteur bezeichnet, welcher vernunftbetont und gleichzeitig eigeninteressiert handelt und auf Basis vollständiger Information seinen eigenen Nutzen maximiert. Seine „Konstruktion“ verdankt er der klassischen und neoklassischen Wirtschaftstheorie. Sie soll vorhersagen, wie sich Klienten oder auch Akteure unter Marktbedingungen verhalten, verhalten werden oder zumindest verhalten sollten. Die Erstbeschreibung des „economic man“ wird dem irischen Dichter und Nationalökonomen John Kells Ingram zugeschrieben [1]. Ingram wiederum bezieht sich auf den Schotten Adam Smith und sein zentrales Werk „The Wealth of Nations“ [2].

Adam Smith, von seiner wissenschaftlichen Herkunft Moralphilosoph, entwickelte die Idee eines im wohlverstandenen Eigeninteresse agierenden, den privaten Nutzen ohne besondere Rücksicht auf das Allgemeingut oder die Interessen anderer verfolgenden Menschen im festen Gottvertrauen, dass durch das Wirken einer „unsichtbar ordnenden Hand" alle diese Eigeninteressen letztlich mit dem Allgemeinwohl zusammenfallen. Ob dem tatsächlich so ist? Ungeachtet der Frage nach der Existenz oder Nicht-Existenz eines Homo oeconomicus in seiner reinen Form hat sich dieses gedankliche Modell in den letzten 200 Jahren evolutiv erfolgreich durchgesetzt. Es ist zwischenzeitlich auch in der Management„Neusprache“ des Gesundheitswesens angekommen, wo es als „Kunde“ oder „Nutzer“ den „Patienten “ zumindest sprachlich in manchen Diskussionen verdrängt hat.

Sicher scheint, dass beginnend mit dem amerikanischen „consumerism“ und der Stärkung der Verbraucherperspektive im marktwirtschaftlichen Geschehen auch das Gesundheitswesen eine sich wandelnde Rolle des Individuums erlebt [3]. Dabei beschränkt sich die Rolle des Individuums innerhalb des „Systems Gesundheitswesen“ nicht nur auf die des Leidenden („Patienten“). Das Individuum ist auch souveräner Nutzer verschiedener präventiver Angebote bei voller Gesundheit, er ist Beitragszahler bei von ihm gewählten Versicherungen und er gestaltet als Bürger die gesamtgesellschaftlichen Rahmenbedingungen verantwortlich mit.

Modernes bürgerschaftliches Engagement betont die Übernahme aktiver, gestaltender öffentlicher Verantwortlichkeit und geht über den bloßen Besitz von Bürgerrechten in einem passiven Sinn hinaus. Bürgerliches und öffentliches Interesse fallen in Hinblick auf die faire Nutzung öffentlicher Güter und Dienstleistungen zusammen. Verantwortliches Handeln wiederum setzt das Vorhandensein von Wahlmöglichkeiten (choices) und ebenso die Möglichkeit zur Artikulation der eigenen Position (voice) voraus. Die Befähigung von Individuen (empowerment) und die Zuweisung von Rechten innerhalb des Gesundheitswesens kann sowohl aus der Perspektive des Patienten als Leistungsempfänger betrachtet werden, als auch aus der Perspektive des Versicherten als Financier der Leistungserbringung. Damit sind grundsätzlich sowohl inter- als auch intra-individuelle Rollenkonflikte angelegt: Die Interessen als Nutzer von Gesundheitsleistungen können durchaus andere sein als die Interessen desjenigen, welcher die Finanzierung über seine Pflichtbeiträge sicherstellt. So ist es vorstellbar, dass dieselbe Person Dienstleistungen des Gesundheitswesens in Anspruch nehmen möchte, z.B. komplementärmedizinische Behandlungsmethoden, welcher sie sich bei voller Gesundheit unter Aspekten der Beitragsbezahlung verschlossen hat. Diese Konflikte auf der Mikroebene lassen sich auch auf der Mesoebene der Institutionen im Gesundheitswesen wie auch auf der Makroebene der Sozialgesetzbücher fortsetzen. Vielleicht liegt in diesen Konflikten die Notwendigkeit begründet, vielfältige Regulierungen und Balancen zu treffen bzw. anzustreben. Im bundesdeutschen korporatistischen Bismarck-System wird für die dafür notwendigen Debatten bzw. Diskussionen und Konsensfindungsprozesse ein Regelwerk vorgehalten, welches dem Individuum zumindest grundsätzlich Beteiligungsmöglichkeiten z.B. über Sozialwahlen oder über die Mitarbeit in Gremien ermöglicht.

Angesichts der bestehenden vielfältigen Regelungen, Restriktionen und Inzentivierungen ist die mancherorts befürwortete Umwandlung des bestehenden Gesundheitssystems in ein marktorientiertes Gesundheitswesen und des Patienten in einen aktiven Verbraucher von Hoffnungen und Befürchtungen begleitet [4]. Aus ethischer Perspektive haben die Grundelemente des Homo oeconomicus durchaus interessante Aspekte. Das Thema der freien Wahl trägt Bezüge zu dem grundlegenden bioethischen Prinzip der Patientenautonomie [5]. Elementare Bestandteile einer autonomen Entscheidung sind die Handlungsfreiheit, die Möglichkeit zur wirksamen Abwägung der Optionen, eine unbeeinflusste Authentizität und die Reflexion anhand der eigenen moralischen Maßstäbe. Handlungsfreiheit wird im 
Gesundheitswesen in jüngster Zeit zunehmend gewährleistet: Realisiert ist die Freiheit zur freien Arztwahl, die Freiheit zur Wahl des Versicherers, die informationelle Selbstbestimmung und anderes mehr. Gleichermaßen wird vielfach eine umfassende Information im Krankheitsfall gesucht, was durch die im Internet verfügbaren vielfältigen Informationsquellen teils erleichtert wird, bei näherem Hinsehen unter Qualitätsaspekten teilweise auch erschwert ist. Häufig ist es auch eine rationale und freie Entscheidung, wenn die Informationsbewertung dann dem Arzt anvertraut wird. Dass die vom Patienten getroffenen Wahlen authentisch mit seinen persönlichen Präferenzen und Lebensplänen sind, wird durch Initiativen wie die einer partizipativen Entscheidungsfindung aktiv unterstützt (siehe z.B. http://www.patient-als-partner.de). Die Reflexion unter moralischen Gesichtspunkten ist hierfür nur ein spezieller Anwendungsfall.

Ökonomen wir Gary Becker haben sich in dieser Tradition dem zwischenmenschlich ebenfalls zu beobachtenden altruistischen Verhalten zugewendet [6]. Ein evolutionärer Sieg des Homo oeconomicus? Wohl nicht in dieser Einfachheit. Selbst im engeren Gebiet der Wirtschaft blieb die Erklärung des menschlichen Verhaltens als reine Nutzenmaximierung nie unkritisiert. Die begrenzte Erklärungskraft dieses Ansatzes hat z.B. den Vorwurf des „Modellplatonismus“ (Hans Albert) hervorgebracht, den Vorwurf, eine darauf aufbauende Ökonomie sei gar keine empirische Wissenschaft. Mit der Etablierung einer experimentellen Okonomik musste das eher simple Homo oeconomicus Modell erheblich erweitert werden: durch eine neue Institutionenökonomik, die Transaktionskostentheorie, die Verhaltensökonomik, die Evolutionsökonomik und das weite Feld der Spieltheorie. Dort angesprochene Faktoren wie asymmetrische Informationen und begrenzte Rationalitäten dürften dem empirisch zu beobachtenden Arzt-Patienten-Verhältnis in einer ganzen Reihe von typischen Situationen mehr entsprechen. Die Annahme vollständiger Information und vernunftbasierter rationaler, Nutzen-maximierender Auswahl unter den Optionen ist sehr schön gefärbt. Sie tritt in der allgemeinen Erfahrung angesichts von das Bewusstsein prägenden Schmerzen, lebensbedrohlicher oder als lebensbedrohlich empfundener Perspektiven, organischer Ausfälle und krankheitsbedingter Angstzustände recht schnell in den Hintergrund. Was zumindest von einem Teil der Patienten gesucht wird, ist manchmal auch ein wohlwollender Paternalismus des Therapeuten gegenüber „seinem/ihrem“ Patienten. Doch was, wenn der Arzt ebenfalls dem „Homo oeconomicus“-Modell entspricht und durchgängig seinen Vorteil sucht, z.B. durch unnötige, angebotsinduzierte Nachfragesteigerung [7]? Oder wenn eine Gesellschaft die Versorgungsleistungen nach diesem Leitbild rationiert - wenn das Nutzenkalkül die Grenzen der Humanität bestimmt [8]? Wobei wir wieder beim Homo patiens, dem leidenden Menschen als dem vermutlich schwächsten und Leid-tragenden gesellschaftlichen Glied angelangt wären.

Stellt sich überhaupt die Frage nach diesen Rollen? Empirisch zu beobachten ist ein differenziertes Bild von unterschiedlichen Rollen ein und derselben Person oder auch verschiedener
Personen, die abhängig von Kontext und Gesundheitsstatus die einer Patientin, eines Nutzers oder eines Kunden beinhalten. Letztere Rollen gelten vor allem im sogenannten „Dritten Gesundheitsmarkt“, dem Wellnessmarkt: hier dürften Nutzer- und Kundenorientierung klar im Vordergrund stehen. Doch auch im klassischen Feld der Arzt-Patienten-Interaktion ist unabdingbar die Berücksichtigung der Patientenperspektive angezeigt.

Mit diesen und ähnlichen Themen beschäftigt sich diese Ausgabe unserer Zeitschrift: mit partizipativer Entscheidungsfindung und Patientenpräferenzen in medizinischen Versorgungszentren, mit telefonischem Gesundheitscoaching und der Entwicklung von Gesundheitsinformationen unter Bürgerbeteiligung, mit dem Verschreibeverhalten von Allgemeinmedizinern in Österreich und Dentaltourismus, mit dem Aut-idem-Verbot, mit der Analyse von Sekundärdaten und Kodierungsproblemen in der deutschen Todesursachenstatistik.

Welches Menschenbild hat im Gesundheitswesen nun den Vorrang: Homo oeconomicus oder Homo patiens? Oder sehen wir nicht auch noch mindestens eine dritte Rolle des Patienten als Ko-Produzenten von Gesundheit? Hier sei an die Unterscheidung von ökonomischen Sphären Kenneth Bouldings erinnert, der - jenseits der einseitigen Tauschökonomie des Marktes pointiert formuliert: „Kein vernünftiger Mensch kann wollen, dass seine Tochter einen Homo oeconomicus heiratet!“ [9]. Menschliches Dasein ist vielfältig, die von den Menschen geschaffenen gesellschaftlichen Realitäten sollten im Idealfall in ihrer Differenziertheit dieser Vielfalt Raum geben. Dies ist sowohl aus ökonomischer Perspektive unter Wettbewerbsaspekten sinnvoll, als auch unter humanitären und gesellschaftlichen Überlegungen unter dem Aspekt der Autonomie und der Freiheit wie auch der Bedarfsgerechtigkeit. Vielleicht sollte man bei der Frage nach dem Primat des Homo oeconomicus oder des Homo patiens weniger die Attribute ökonomisch bzw. patiens im Auge behalten, sondern vielmehr das Substantiv Homo - der Mensch.

\section{Literatur}

1 Ingram JK. A history of political economy. Edinburgh, Adam and Charles Black, 1888; URL: http://socserv.mcmaster.ca/econ/ugcm/ 3113/smith/wealth/index.html Zugriff am 07.07.2011

2 Smith $A$. An inquiry into the nature and causes of the wealth of nations. London, Methuen \& Company, Ltd., 1776; URL: http://socserv.mcmaster. $\mathrm{ca} / \mathrm{econ} / \mathrm{ug} \mathrm{cm} / 3113 / \mathrm{smith} /$ wealth/index.html, Zugriff am 07.07.2011

3 Wildner M, den Exter AP, van der Kraan WGM. The changing role of the individual in social health insurance systems. In: Saltman RB, Busse R, Figueras J, Hrsg. Social health insurance systems in western Europe. Maidenhead Open University Press, 2004

4 Hengsbach F. „Mehr Markt“ macht nicht gesund - Gesellschaftliche Risiken und solidarische Sicherung entsprechen einander. Gesundheitswesen 2008; 70: 339-349

5 Beauchamps T, Childress J. Principles of biomedical ethics. New York: Oxford University Press, 1994

6 Becker GS. Die Ökonomik des Alltags. Tübingen: Mohr Siebeck, 1998

7 Sanghavi DM. The perils of excessive medical care. Lancet 2011; 377: 1561-1562

8 Kuhn J. Wenn sich Therapie nicht lohnt: Gesundheit als ökonomisches Optimierungsproblem? FKP 2009; 53: 50-54. URL: http://www. josephkuhn.de/pdf/Gesundheit_Oekonomie.pdf, Zugriff am 08.07.2011

9 Boulding KE. Economics as a moral science. Am Econ Rev 1969; 59 (1): $1-12$ 